\title{
Instabilité microsatellitaire dans le cancer colorectal
}

\author{
Microsatellite instability in colorectal cancer
}

\author{
F. Bibeau \\ (C) Lavoisier SAS 2015
}

Au cours de l'édition 2014 du congrès de l'American Society of Clinical Oncology (ASCO) de nouvelles données concernant l'impact pronostique et prédictif de l'instabilité microsatellitaire dans le cancer colorectal ont été présentées. Rappelons que l'instabilité microsatellitaire (MSI) s'observe dans $15 \%$ des cancers colorectaux et que $2 \%$ des MSI s'inscrivent dans un contexte héréditaire correspondant au syndrome de Lynch.

Le statut MSI est considéré comme un marqueur de bon pronostic dans les cancers colorectaux, mais l'utilisation du 5FU (5-fluorouracile) dans les stades II était jusqu'à maintenant considéré comme délétère [1]. Deux travaux ont été présentés à l'ASCO 2014, permettant d'éclaircir les indications thérapeutiques chez les patients opérés d'un cancer colique MSI.

Une étude française de l'AGEO (Association des Gastroentérologues Oncologues) menée par David Tougeron s'est appuyée sur une série rétrospective de 433 patients opérés, porteurs de cancer colique MSI réséqué, dont 170 ont reçu une chimiothérapie adjuvante (5-FU : $30 \%$; FOLFOX : $70 \%$ [2]. Les résultats montrent qu'une chimiothérapie adjuvante à base de FOLFOX (acide folinique/5fluorouracile/oxalipaltine) semble efficace chez les patients opérés d'une tumeur MSI. Ceci est observé pour les stades III, les données pour les stades II à haut risque étant moins formelles, compte tenu de la faible taille des effectifs. Dans cette série, le traitement par 5FU seul n'apparait pas significatif.

Par ailleurs, une seconde étude, cette fois-ci américaine, reposant sur la base de données ACCENT et comportant 7803 patients traités par 5-FU adjuvant ou chirurgie seule, a été menée par Dan Sargent [3]. Elle a souligné la valeur pronostique favorable du statut MSI pour les cancers de stades II opérés et non traités par chimiothérapie adjuvante, mais pas pour les stades III. Après traitement par 5FU, aucun impact positif ou délétère n'était objectivé pour les cancers

\section{F. Bibeau $(\square)$}

Département de Pathologie, Institut du Cancer de Montpellier, 208, rue des apothicaires, 34298 Montpellier cedex 5

e-mail : frederic.bibeau@icm.unicancer.fr de stades II, alors qu'un effet positif et significatif apparaissait pour les stades III. En conséquence l'auteur concluait, qu'aucun traitement adjuvant ne se justifiait dans les cancers coliques MSI de stade II, compte-tenu de leur excellent pronostic spontané, mais que le statut MSI ne devait en revanche pas être pris en compte pour un traitement adjuvant à base de $5 \mathrm{FU}$ pour les stades III.

\section{En conclusion}

La détermination du statut MSI est utile dans les cancers colorectaux de stade II, car c'est un marqueur de bon pronostic qui rend caduque l'utilisation du 5FU, pour lequel aucun impact délétère ou positif ne semble exister. Elle est indispensable pour les tumeurs de stade II à haut risque pour proposer un éventuel traitement adjuvant qui devrait être du FOLFOX et non du 5FU seul.

La détermination du statut MSI n'est pas utile chez les patients opérés d'un cancer colique de stade III pour décider d'un traitement adjuvant et de son type (FOLFOX ou 5FU).

Ces constatations renforcent le rôle des plateformes d'oncogénétique somatique des tumeurs dans la détermination de ce paramètre moléculaire des cancers colorectaux.

Liens d'intérêts : l'auteur déclare ne pas avoir de liens d'intérêts.

\section{Références}

1. Sargent DJ, Marsoni S, Monges G, et al (2010) Defective mismatch repair as a predictive marker for lack of efficacy of fluorouracil-based adjuvant therapy in colon cancer. J Clin Oncol 28:3219-26

2. Tougeron D, Sickersen G, Lecomte T, et al (2014) Impact of adjuvant chemotherapy with 5-FU or FOLFOX in colon cancers with microsatellite instability: an AGEO multicenter study. J Clin Oncol 32:5s (suppl abstr 3508)

3. Sargent DJ, Shi Q, Yothers G, et al (2014) Prognostic impact of deficient mismatch repair in 7.803 stageII/III colon cancer patients: A pooled individual patient data analysis of 17 adjuvant trials from the ACCENT database. J Clin Oncol 32:5s (suppl; abstr 3507) 